\title{
El parasitismo por Profilicollis bullocki (Acanthocephala: Polymorphidae) en Emerita analoga (Anomura: Hippidae) según condiciones contrastantes de abundancia de hospedadores definitivos en Chile
}

Parasitism by Profilicollis bullocki (Acanthocephala: Polymorphidae) in Emerita analoga (Anomura, Hippidae), according to contrasting conditions of abundance of definitive hosts in Chile

\section{David Zambrano ${ }^{1}$ y Mario George-Nascimento ${ }^{1}$}

\begin{abstract}
${ }^{1}$ Departamento de Ecología Costera, Facultad de Ciencias, Universidad Católica de la Santísima Concepción, Casilla 297, Concepción, Chile. mgeorgen@ucsc.cl
\end{abstract}

\begin{abstract}
In Chile, the intermediate host of the acanthocephalan Profilicollis bullocki is the mole crab Emerita analoga, with seabirds as the definitive hosts, who arrive in mass during summer, enhancing the spread of acanthocephalan eggs to the environment. Thus, the magnitude of this parasitosis in $E$. analoga should attain higher values after the yearly arrival of seabirds. This study was performed in two periods contrasting in the abundance of marine birds, SeptemberNovember 2008 (late winter-spring) and December 2008-January 2009 (summer), at 3 sandy beaches of the Biobío region, Chile. The abundance of birds was highest in summer, mainly due to the arrival of the Franklin 's gull Larus pipixcan to the study sites. It was observed that the cephalotorax length (LCT) of $E$. analoga was much smaller in summer than in spring, and that the prevalence and abundance of $P$. bullocki increased with LCT. Although the prevalence of $P$. bullocki was higher before the arrival of birds, the slopes of the linear regressions between the abundance and intensity with LCT were higher after the arrival of the birds to the study sites. These results are partly explained by the comparatively large LCT of mole crabs before the arrival of seabirds, and by an intense recruitment pulse of small $E$. analoga after the arrival of seabirds, thus provoking a dilution effect on the prevalence.
\end{abstract}

Key words: Prevalence, intensity, seabirds, cystacanths

\begin{abstract}
Resumen.- En Chile, el hospedador intermediario del acantocéfalo Profilicollis bullocki es el crustáceo Emerita analoga y varias especies de aves marinas son sus hospedadores definitivos, las cuales llegan masivamente a las playas en primavera-verano, luego de migraciones entre hemisferios, potenciando la propagación de huevos del acantocéfalo al medio. Por esto, la magnitud de esta parasitosis debiera aumentar luego del arribo anual de aves marinas migratorias. El presente estudio se realizó en dos períodos contrastantes en la abundancia de aves marinas, septiembre-noviembre de 2008 (invierno tardío-primavera) y diciembre 2008-enero de 2009 (verano), en 3 playas arenosas de la región del Biobío, Chile. Se encontró mayor abundancia de aves en el verano, principalmente por la llegada de la gaviota de Franklin Larus pipixcan a los sitios de estudio. En las muestras de $E$. analoga examinadas se observó que la longitud cefalotoráxica (LCT) de los ejemplares muestreados en verano era mucho menor que en otoño-invierno, y que la prevalencia y abundancia de $P$. bullocki aumentaban con la LCT de $E$. analoga, aunque la abundancia y la intensidad mostraron mayores pendientes, luego del arribo de aves a los sitios de estudio. Estos resultados estarían influenciados por la mayor LCT promedio de E. analoga en el período previo al arribo de las aves, y por el efecto de dilución que provocó el reclutamiento de $E$. analoga sobre los descriptores cuantitativos del parasitismo.
\end{abstract}

Palabras clave: Prevalencia, intensidad, aves marinas, cistacanto

\section{INTRODUCCIÓN}

Cuando el ciclo de vida de los parásitos es indirecto, su abundancia depende de la presencia y de la abundancia de sus hospedadores intermediarios y definitivos, y por ende de la frecuencia de encuentro entre ellos (Combes 1995). Profilicollis bullocki Mateo, Córdova \& Guzmán, 1982, es un acantocéfalo que como todas las especies del phylum, posee un ciclo de vida indirecto, caracterizado por el uso de artrópodos como hospedadores intermediarios (Nickol et al. 2002, Kennedy 2006). A su vez estos crustáceos son presas de varias especies de aves marinas que actúan como sus hospedadores definitivos (Crompton \& Nickol 1985), y existen registros de P. bullocki en mamíferos (Hennessy \& Morejohn 1977) incluido el hombre (Tantaleán et al. 2002). Esta especie de acantocéfalo 
utiliza como hospedador intermediario a Emerita analoga (Stimpson, 1857), donde habita al estado de cistacanto en el hemocele, y alcanza el estado adulto en el intestino de aves marinas como Larus pipixcan, Larus dominicanus, Larus maculipennis, Larus modestus, Larus serranus, Phalacrocorax atriceps y Calidris spp. (Hinojosa-Sáez \& González-Acuña 2005, Riquelme et al. 2006, Balboa et al. 2009). Una vez que el ave ingiere al crustáceo infectado y que los parásitos alcanzan su estado adulto, las hembras ovígeras liberan los huevos en el intestino del hospedador y a través de las heces son diseminados al ambiente. En el medio acuático los huevos son filtrados por E. analoga, desarrollándose el estado juvenil de cistacanto enquistado en la cavidad celómica del crustáceo, aunque se desconoce el tiempo que el parásito precisa para alcanzar el estado de cistacanto dentro del crustáceo.

Emerita analoga es un crustáceo característico de las zonas intermareal e infralitoral de las playas de arena de las costas del Pacífico de América y a la vez, es un eslabón importante en las tramas tróficas de este sistema (Oliva et al. 1992, Contreras et al. 2000). Su distribución geográfica se extiende desde Alaska, EE.UU. $\left(58^{\circ} \mathrm{N}\right)$ hasta Aysén, Chile $\left(55^{\circ} \mathrm{S}\right)$, exceptuando las zonas tropicales de aguas con temperaturas superiores a los $20^{\circ} \mathrm{C}$ (Contreras et al. 1999). Este crustáceo vive alrededor de 3 a 4 años (Contreras et al. 2000) y aunque es posible encontrar juveniles durante todo el año, posee máximos de reclutamiento en primavera-verano (Lépez et al. 2001). Recientes avances en la biología reproductiva de las especies de este género señalan que son hermafroditas protándricos (Subramoniam \& Gunamalai 2003). Entre los parásitos descritos para $E$. analoga en el océano Pacífico sudoriental (costa de Perú y Chile), se incluyen formas larvales del acantocéfalo $P$. bullocki, del digeneo Maritrema sp., de los eucéstodos Nybelinia sp. y Eutetrarhynchus sp., y del nemátodo Proleptus sp. (Oliva et al. 1992, Iannacone et al. 2007, Balboa et al. 2009). En general, las infecciones por estados larvales de parásitos son acumulativas, y en consecuencia, su abundancia aumenta con el tamaño corporal del hospedador (Alvitres et al. 1999, Smith 2007, Oliva et al. 2008), aunque esta correlación no ha sido encontrada en todos los estudios realizados (Iannacone et al. 2007).

Los hospedadores definitivos de $P$. bullocki poseen una amplia distribución geográfica, tanto en Sudamérica como en otros sitios del mundo (Bahamondes \& Castilla 1986). En consecuencia, dispersan los huevos del parásito en un amplio rango geográfico. Este aporte es potencialmente mayor cuando las aves marinas llegan masivamente a las playas en ciertos períodos estacionales, como parte de su migración anual (Smith 2007). Por ello, una gran cantidad de aves marinas conduciría a una mayor abundancia de $P$. bullocki, lo que sugiere que en primavera-verano, cuando arriban masivamente a la zona en estudio, debería haber mayor abundancia de este acantocéfalo que en otoñoinvierno.

Los objetivos de este estudio fueron evaluar: 1) si la abundancia de $P$. bullocki aumenta con el tamaño corporal de $E$. analoga y 2) si la prevalencia, abundancia e intensidad de $P$. bullocki en E. analoga es menor antes de la llegada masiva de aves marinas que después de su llegada. Para ello, se determinó la relación entre la longitud corporal de $E$. analoga con la prevalencia, abundancia e intensidad de $P$. bullocki. Además, se comparó la abundancia, prevalencia e intensidad de $P$. bullocki en E. analoga antes y después de la llegada anual de aves marinas a los sitios de estudio.

\section{Material Y MÉTOdoS}

El estudio se realizó en tres playas arenosas de la región del Biobío, Chile. Las localidades fueron: Playa Colcura $\left(37^{\circ} 06^{\prime} \mathrm{S}, 73^{\circ} 08^{\prime} \mathrm{W}\right)$, Playa Blanca (37'03'S, 73'08'W) y Caleta Lenga (36'45'S, 73'10'W).

Para registrar la abundancia de aves, cada localidad fue visitada en tres ocasiones antes de la llegada de las aves migratorias (entre el 7 de de septiembre y el 3 de noviembre de 2008) y en tres oportunidades después de su llegada (entre el 5 de diciembre de 2008 y el 5 de enero de 2009). En cada visita se procedió a identificar (Araya \& Millie 2005) y contar in situ o en placas fotográficas (9 a 10 veces por día) las especies de aves marinas presentes en tres direcciones distintas de la costa (izquierda, frente y derecha) y en 3 momentos del día (mañana, mediodía y tarde). Los recuentos de aves abarcaban un radio aproximado de $60 \mathrm{~m}$ desde el sitio de observación. El promedio de estos conteos se consideró como un estimador puntual de lo que sucedía en cada período.

Para evaluar el parasitismo en E. analoga en cada una de las tres localidades se realizaron dos muestreos. El primer muestreo fue realizado a principios de septiembre de 2008 (a fines de invierno y comienzo de primavera), antes de la llegada masiva de aves migratorias. La segunda muestra se tomó en los meses de diciembre 2008 y enero de 2009 (verano), después de la llegada masiva de aves migratorias. Los muestreos del crustáceo se realizaron en las mismas fechas de evaluación de la abundancia de aves. El área determinada para la toma de las muestras de E. analoga correspondió a la zona espejo de la zona 
intermareal, a lo largo de transectos paralelos a la costa. Se recolectaron manualmente 392 ejemplares de E. analoga durante las mareas bajas, los cuales fueron guardados en bolsas plásticas y congelados para su posterior examen en el laboratorio.

Una vez en el laboratorio, se midió la LCT de cada ejemplar de E. analoga con un pie de metro de 0,1 $\mathrm{mm}$ de sensibilidad. Para determinar la presencia de $P$. bullocki, los ejemplares fueron disectados removiendo el caparazón y el hemocele, examinando sus órganos bajo lupa estereoscópica. Se determinaron los siguientes descriptores cuantitativos del parasitismo: prevalencia (porcentaje de hospedadores infectados), abundancia (promedio de parásitos por hospedador examinado) e intensidad media (promedio de parásitos por hospedador parasitado, ver Margolis et al. 1982).

Para evaluar diferencias significativas en la cantidad de aves marinas entre septiembre-noviembre y diciembreenero, se aplicó un análisis de la varianza de 3 vías, en que el período de muestreo, la localidad de muestreo y la especie de ave fueron considerados factores fijos, con 2 , 3 y 4 niveles respectivamente. Los datos de avistamiento total de aves, por especie, por día y por localidad, fueron transformados al $\log _{10}(\mathrm{x}+1)$ para cumplir con el requisito de homogeneidad de varianzas, luego de ser evaluados con la prueba de Cochran (Zar 1984).

La significancia estadística de las variaciones en LCT de E. analoga según período de muestreo se determinó con un análisis de la varianza de una vía. Se usaron tablas de contingencia toda vez que se compararon prevalencias, las que fueron evaluadas mediante $\chi^{2}$ (Agresti 1996).

La relación de la prevalencia, de la abundancia y de la intensidad del parasitismo con la LCT de E. analoga fue evaluada con el coeficiente de correlación de Pearson. El hallazgo de coeficientes positivos y significativos permitiría inferir su acumulación a lo largo de la ontogenia del hospedador. Se emplearon análisis de la covarianza de la abundancia e intensidad de $P$. bullocki en los que la LCT de E. analoga era la covariable, el período previo y posterior a la llegada masiva de aves marinas era uno de los factores (Zar 1984). Los datos de la abundancia de $P$. bullocki fueron transformados al $\log _{10}(\mathrm{x}+1)$ y los de la intensidad al $\log _{10}(\mathrm{x})$ con el objeto de hacer más lineal su relación con la LCT.

\section{Resultados}

De las 4 especies de aves marinas observadas, Larus dominicanus y Phalacrocorax $\mathrm{sp}$. fueron especies comunes, aunque no muy abundantes en ambos períodos de muestreo, en tanto que en diciembre-enero, la abundancia de Numenius phaeopus disminuyó fuertemente mientras que la de Larus pipixcan aumentó (Tabla 1). El factor que mejor explicaba la variación de la abundancia de aves fue la interacción entre el período de muestreo y la identidad específica de las aves $\left(\mathrm{F}_{(3,215)}=231,2, P<0,0001\right.$, Tabla 2). La interacción entre el período de muestreo y la localidad de muestreo fue el único factor que mostró no tener un efecto significativo sobre la variación de la abundancia de aves $\left(\mathrm{F}_{(2,215)}=0,13 ; 0,90>P>0,80\right)$. Los demás factores, aunque significativos, explican una pequeña fracción de la varianza de la abundancia de aves (Tabla 2).

La LCT de E. analoga fue significativamente mayor en las muestras tomadas antes de la llegada de aves marinas $\left(\mathrm{F}_{(1,390)}=14,83, P<0,05\right.$, Tabla 3$)$.

En el 47,2\% de los 392 ejemplares de E. analoga se encontraron cistacantos de $P$. bullocki (Tabla 3). La prevalencia $\left(\chi^{2}=87,3\right.$, g. $1 .=1, P<0,001$, Tabla $3)$, y la abundancia $\left(\mathrm{F}_{(1,390)}=49,77\right.$, Tabla 3) fueron significativamente mayores antes de la llegada de las aves. Sin embargo, la intensidad fue significativamente mayor

Tabla 1. Número de observaciones por playa $(n)$ y abundancia promedio $\log _{10}(x+1) \pm$ d.e. de cuatro especies de aves marinas registradas en tres playas de la región del Biobío, Chile, en dos periodos de muestreo / Number of observations per beach $(\mathrm{n})$ and mean abundance $\pm \mathrm{s}$.d. $\log _{10}(x+1)$ of four seabird species recorded at three beaches in the Biobío region, Chile, in two sampling periods

\begin{tabular}{|c|c|c|c|c|c|c|}
\hline \multirow[b]{2}{*}{ Aves marinas } & \multicolumn{3}{|c|}{ Septiembre-Noviembre } & \multicolumn{3}{|c|}{ Diciembre-Enero } \\
\hline & $\begin{array}{c}\text { Playa Colcura } \\
n=9\end{array}$ & $\begin{array}{c}\text { Playa Blanca } \\
n=10\end{array}$ & $\begin{array}{c}\text { Playa Lenga } \\
n=10\end{array}$ & $\begin{array}{c}\text { Playa Colcura } \\
n=10\end{array}$ & $\begin{array}{c}\text { Playa Blanca } \\
n=10\end{array}$ & $\begin{array}{c}\text { Playa Lenga } \\
\qquad n=10\end{array}$ \\
\hline Larus pipixcan & $0 \pm 0$ & $0 \pm 0$ & $0 \pm 0$ & $1,11 \pm 0,09$ & $0,94 \pm 0,17$ & $0,91 \pm 0,18$ \\
\hline Larus dominicanus & $0,84 \pm 0,11$ & $0,73 \pm 0,24$ & $0,77 \pm 0,21$ & $1,02 \pm 0,06$ & $0,87 \pm 0,13$ & $1,71 \pm 0,19$ \\
\hline Phalacrocorax sp. & $0,67 \pm 0,08$ & $0,73 \pm 0,16$ & $0,54 \pm 0,30$ & $0,88 \pm 0,07$ & $0,82 \pm 0,09$ & $0,61 \pm 0,27$ \\
\hline Numenius phaeopus & $1,16 \pm 0,10$ & $0.82 \pm 0,19$ & $0,91 \pm 0,28$ & $0,68 \pm 0,06$ & $0,75 \pm 0,12$ & $0 \pm 0$ \\
\hline
\end{tabular}


Tabla 2. Análisis de la varianza de la abundancia de aves marinas según período, playa y especie. Datos transformados a $\log 10(x+1)$. $(g l=$ grados de libertad; $S C=$ suma de cuadrados; $C M=$ cuadrados medios; $F=$ valor de estadígrafo $\mathrm{F}$ de Fisher; $\mathrm{P}=$ probabilidad) / Analysis of the variance of the abundance of seabirds according to the sampling period, beach and species. Data were $\log 10(\mathrm{x}+1)$ transformed $(\mathrm{gl}=$ degrees of freedom; $\mathrm{SC}=\mathrm{sum}$ of squares; $C M=$ Mean squares; $F=F$ value of Fisher's statistic; $P$ = probability)

\begin{tabular}{lrrrrr}
\hline \multicolumn{1}{c}{ Fuente de variación } & gl & SC & CM & F & $P$ \\
\hline Llegada de aves marinas & 1 & 4,08589 & 4,08589 & 167,26 & $<0,001$ \\
Playa & 2 & 0,54239 & 0,27119 & 11,10 & $<0,001$ \\
Especie & 3 & 7,44167 & 2,48056 & 101,54 & $<0,001$ \\
Llegada de aves marinas x Playa & 2 & 0,00627 & 0,00314 & 0,13 & 0,879 \\
Llegada de aves marinas x Especie & 3 & 16,94193 & 5,64731 & 231,17 & $<0,001$ \\
Playa x Especie & 6 & 4,42057 & 0,73676 & 30,16 & $<0,001$ \\
Llegada de aves marinas x Playa x Especie & 6 & 3,95747 & 0,65958 & 27,00 & $<0,001$ \\
& & & & & \\
Error & 215 & 5,2522 & 0,02443 & & \\
Total & 238 & 42,6484 & & & \\
\hline
\end{tabular}

Tabla 3. Número de ejemplares de $E$. analoga de 5 clases de longitud cefalotoráxica (LCT) que se encontraron con y $\sin P$. bullocki, antes y después de la llegada de aves al área de estudio / Number of $E$. analoga specimens in 5 classes of the cephalothorax length (LCT), found with and without $P$. bullocki, before and after the arrival of seabirds to the study area

\begin{tabular}{|c|c|c|c|c|}
\hline \multirow[b]{2}{*}{ Clases de LCT (mm) } & \multicolumn{2}{|c|}{ Antes } & \multicolumn{2}{|c|}{ Después } \\
\hline & Parasitados & No parasitados & Parasitados & No parasitados \\
\hline $7,0-12,0$ & 11 & 20 & 0 & 114 \\
\hline $12,1-17,0$ & 61 & 18 & 30 & 43 \\
\hline $17,1-22,0$ & 41 & 4 & 6 & 4 \\
\hline $22,1-27,0$ & 9 & 1 & 14 & 3 \\
\hline $27,1-32,0$ & 3 & 0 & 10 & 0 \\
\hline Total & 125 & 43 & 60 & 164 \\
\hline
\end{tabular}

después de la llegada de las aves $\left(\mathrm{F}_{(1,183)}=4,54, P<0,04\right.$, Tabla 4).

La prevalencia aumentó con la LCT de E. analoga (r $=0,91, \mathrm{n}=5$ intervalos de LCT, $P<0,05$, Tabla 3$)$. Al comparar entre hospedadores de similar LCT (entre 12,1 y 22,0 mm), se encontró que la prevalencia fue mayor antes de la llegada de las aves $\left(\chi^{2}=32,1\right.$, g. $1 .=1, P<0,001$, Tabla 3).

La abundancia de $P$. bullocki resultó positiva y significativamente correlacionada con la LCT de $E$. analoga $\left(\mathrm{r}=0,45, \mathrm{~F}_{(1,390)}=310,5, P<0,001\right)$. El análisis de la covarianza de la abundancia de $P$. bullocki mostró que aumentaba de manera distinta para ambos períodos $\left(\mathrm{F}_{(1,}\right.$ $\left.{ }_{388)}=17,13, P<0,001\right)$, ya que la pendiente de la regresión entre ambas variables fue mayor después del arribo de las aves (pendiente \pm 1 .e.e. $=0,0592 \pm 0,0031, \mathrm{R}^{2}=0,623 ; \mathrm{n}=$ $168, P<0,001)$ que antes de su llegada (pendiente $\pm 1 . e . e=$
$0,0343 \pm 0,0056, \mathrm{R}^{2}=0,184, \mathrm{n}=224, P<0,001$, Fig. 1$)$. El análisis de la covarianza de la intensidad también mostró que aumentaba de forma distinta en ambos períodos $\left(\mathrm{F}_{(1,}\right.$ $\left.{ }_{181)}=10,01, P=0,0018\right)$, ya que se halló una pendiente mayor en el período posterior a la llegada de las aves (pendiente \pm 1 e. e. $=0,0530 \pm 0,0081, \mathrm{R}^{2}=0,423, \mathrm{n}=60$, $P<0,001)$ fue significativamente mayor que en el período previo (pendiente \pm 1 e. e. $=0,0192 \pm 0,00715, \mathrm{R}^{2}=0,056$, $\mathrm{n}=125, P<0,008)$.

\section{Discusión}

Las aves marinas, como hospedadores definitivos, contribuyen a la dispersión de sus parásitos en el rango de su distribución geográfica, transportando sus parásitos sobre considerables distancias (Smith 2001), como consecuencia de su migración interhemisférica. Este mecanismo ha sido descrito para Polymorphus kenti (Iannacone et al. 2007), 
Tabla 4. Prevalencia (P, \%), intensidad y abundancia (promedio y desviación estándar entre paréntesis) del parasitismo por $P$. bullocki en 392 ejemplares de $E$. analoga según el período de muestreo (antes y después de la llegadas de aves marinas a los sitios de estudio) / Prevalence (P, \%), abundance and intensity (mean and standard deviation in parenthesis) of parasitism by $P$. bullocki in 392 E. analoga specimens, according to sampling period (before and after the arrival of seabirds to the study sites)

\begin{tabular}{lccc}
\hline Período & Prevalencia & Intensidad & Abundancia \\
\hline Antes & 74,4 & $0,477(0,347)$ & $0,466(0,358)$ \\
Después & 26,8 & $0,608(0,467)$ & $0,199(0,380)$ \\
Total & 47,2 & $0,519(0,393)$ & $0,313(0,393)$ \\
\hline
\end{tabular}

y es el que se propone para la dispersión geográfica de P. bullocki. Sin embargo, la validez taxonómica de varias especies del género Profilicollis (= Polymorphus) aún se encuentran en revisión (Mateo et al. 1983, García-Varela \& Pérez-Ponce de León 2008, Balboa et al. 2009), por lo que ambas podrían ser conespecíficas.

Los resultados de las variaciones de la abundancia de aves muestran que la elección de los dos períodos de muestreo fue adecuada para contrastar su potencial efecto sobre la abundancia de $P$. bullocki, ya que las especies migratorias resultaron ser más abundantes en el período estival (Tablas 1 y 2). Por otra parte, hay estudios que respaldan una asociación positiva entre la abundancia de las aves con la prevalencia y la abundancia de parásitos en E. analoga en playas arenosas de California (Smith 2007) y del norte de Chile (Oliva et al. 2008). Esto resalta la importancia de la abundancia de los hospedadores en la transmisión de los parásitos, ya que los patrones de visita y distribución de los hospedadores definitivos podrían crear una variación espacial y temporal en la prevalencia y abundancia del parásito en el hospedador intermediario (Riquelme et al. 2006, Smith 2007, Oliva et al. 2008).

Sin embargo, en este estudio los resultados obtenidos no fueron lo que se esperaba ya que la prevalencia total y la abundancia total de $P$. bullocki resultaron mayores antes de la llegada de las aves (Tabla 3). Esto podría explicarse por el incremento de la abundancia relativa en ejemplares de $E$. analoga cuya LCT era menor a $12 \mathrm{~mm}$ en verano, debido a un intenso pulso de reclutamiento de este crustáceo ocurrido en el verano (Tabla 3), ocasionando no solamente una disminución de la LCT promedio sino que también la disminución en la prevalencia total. Esta observación permite resaltar la necesidad de realizar comparaciones de la prevalencia, abundancia e intensidad entre hospedadores de similar tamaño corporal. Sin embargo, aunque esta diferencia debiera desaparecer al comparar ejemplares de similar LCT recolectados en ambos períodos (entre 12,1 y $22 \mathrm{~mm}$ de LCT, Tabla 3), la prevalencia siguió siendo mayor antes de la llegada de las aves. Aunque no tenemos una explicación a este resultado, la mayor pendiente de la regresión entre la abundancia y la intensidad de $P$. bullock $i$ con la LCT de E. analoga, después de la llegada de las aves (Fig. 1), refuerza la hipótesis de que la llegada de las aves provoca un aumento del parasitismo por P. bullocki en E. analoga.

Aunque la diferencia en la LCT entre los períodos de muestreo habría sido producto de los ciclos naturales de reclutamiento de E. analoga (Dugan et al. 1994), destacamos que en este estudio hubo un esfuerzo especial en muestrear ejemplares menores a $18 \mathrm{~mm}$ de longitud cefalotóraxica (LCT) antes de la llegada de las aves. Este procedimiento habría permitido maximizar la probabilidad de hallar ejemplares no parasitados en las muestras y detectar si la prevalencia del parasitismo se modifica significativamente con la llegada estacional de aves marinas migratorias. Este esfuerzo, sin embargo, no fue fructífero pues la abundancia de crustáceos pequeños era escasa a fines del otoño de 2008. En suma, habría existido una cohorte de $E$. analoga que comenzó a reclutar a principios de enero de 2008 (Lépez et al. 2001), lo que explicaría la baja prevalencia total observada en el período

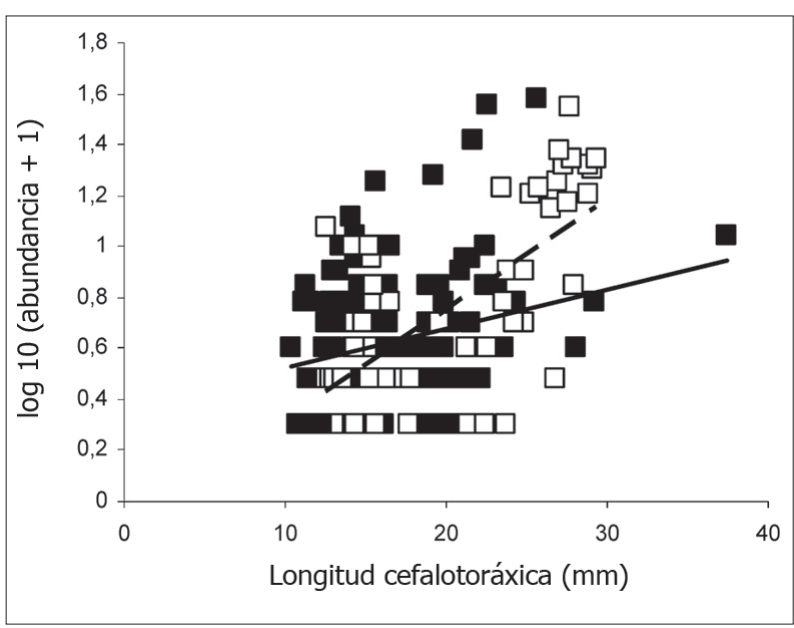

Figura 1. Relación entre la abundancia de $P$. bullocki y la longitud cefalotoráxica $(\mathrm{mm})$ de $E$. analoga, antes $(\mathrm{n}=168$, línea continua, cuadrados negros) y después ( $\mathrm{n}=224$, línea discontinua, cuadrados blancos) de la llegada masiva de aves marinas al área de estudio / Relationship between the abundance of $P$. bullock $i$ and the length of the cephalothorax $(\mathrm{mm})$ in $E$. analoga, before $(\mathrm{n}=168$, continuous line, black squares) and after $(n=224$, dashed line, white squares) the arrival of seabirds to the study area 
después de la llegada masiva de aves, con un menor tiempo para la transmisión de los huevos del acantocéfalo al hospedador intermediario.

Otros factores que podrían ser relevantes en la transmisión del acantocéfalo y que afectan la probabilidad de encuentro entre los huevos del parásito y el crustáceo, no fueron considerados en este estudio, como son el movimiento de las mareas, la fuerza del oleaje (Latham \& Poulin 2003), el tipo de playa (disipativa o reflectiva, Jaramillo et al. 2000), y el patrón de visitas que las aves realizan a las distintas playas. En conclusión, la abundancia de cistacantos en E. analoga no sólo está asociada al tamaño corporal de su hospedador intermediario, sino que también a la llegada anual de los hospedadores definitivos y a la dinámica de la población del crustáceo. Esta última podría modificar la abundancia relativa de las clases de tamaño del hospedador intermediario y afectar el valor que alcanzan algunos descriptores cuantitativos del parasitismo, incluso entre playas cercanas. Por ello, futuros protocolos de análisis de la información en este tipo de sistemas han de contemplar estos aspectos.

\section{Agradecimientos}

Se agradecen los comentarios críticos de dos evaluadores anónimos que permitieron mejorar sustantivamente la versión inicial de este manuscrito.

\section{LiTERATURA CITADA}

Agresti A. 1996. An introduction to categorical data analysis, 290 pp. John Wiley \& Sons, London.

Alvitres V, J Chanamé, J Fupuy, R Chambergo \& M Cortéz. 1999. Cambios en la prevalencia de los helmintos parásitos de Emerita analoga por efecto de 'El Niño 1997'98‘. Revista Peruana de Biología, Volumen Extraordinario: 69-75.

Araya BM \& G Millie. 2005. Guía de campo de las aves de Chile, 410 pp. Editorial Universitaria, Santiago.

Bahamondes I \& JC Castilla. 1986. Predation of marine invertebrates by kelp gull Larus dominicanus in undisturbed intertidal rocky shores of central Chile. Revista Chilena de Historia Natural 59: 65-72.

Balboa L, A Hinojosa, C Riquelme, S Rodríguez, J Bustos \& M George-Nascimento. 2009. Alloxenic distribution of cystacanths of two species of Profilicollis in sympatric crustacean species in Chile. Journal of Parasitology 95: 1205-1208.

Combes C. 1995. Interactions durables. Écologie et évolution du parasitisme, 524 pp. Masson, Paris.
Contreras H, O Defeo \& E Jaramillo. 1999. Life history of Emerita analoga (Stimpson) (Anomura, Hippidae) in a sandy beach of south central Chile. Estuarine, Coastal and Shelf Science 48: 101-112.

Contreras H, E Jaramillo \& P Quijón. 2000. Natural history of Emerita analoga (Stimpson) (Anomura, Hippidae) in a sandy beach of northern Chile. Revista Chilena de Historia Natural 73: 705-715.

Crompton D \& B Nickol. 1985. Biology of the Acanthocephala, 518 pp. Cambridge University Press, Cambridge.

Dugan J, EM Hubbard \& AM Wenner. 1994. Geographic variation in the reproductive biology of the sand crab, Emerita analoga (Stimpson) on the California coast: relationships to environmental variables. Journal of Experimental Marine Biology and Ecology 181: 255-278.

García-Varela M \& G Pérez-Ponce de León. 2008. Validating the systematic position of Profilicollis Meyer, 1931 and Hexaglandula Petrochenko, 1950 (Acanthocephala: Polymorphidae) using cytochrome $\mathrm{C}$ oxidase (cox 1). Journal of Parasitology 94: 212-217.

Hennessy H \& V Morejohn. 1977. Acanthocephalan parasites of the sea otter, Enhydra lutris, off coastal California. California Fish \& Game 63: 268-272.

Hinojosa-Sáez A \& D González-Acuña. 2005. Estado actual del conocimiento de helmintos en aves silvestres de Chile. Gayana 69: 241-253.

Iannacone J, L Alvariño \& B Bolognesi. 2007. Aspectos cuantitativos de los metazoos parásitos del muy muy Emerita analoga (Stimpson) (Decapoda, Hippidae) en Chorrillos, Lima, Perú. Neotropical Helminthology 1: 59-67.

Jaramillo E, J Dugan \& H Contreras. 2000. Abundance, tidal movement, population structure and burrowing rate of Emerita analoga (Anomura, Hippidae) at dissipative and reflective sandy beach in south central Chile. Marine Ecology 21: 113-127.

Kennedy CR. 2006. Ecology of the Acanthocephala, 249 pp. Cambridge University Press, Cambridge.

Latham ADM \& R Poulin. 2003. Spatiotemporal heterogeneity in recruitment of larval parasite to shore crab intermediate hosts: The influence of shorebird definitive hosts. Canadian Journal of Zoology 81: 1282-1291.

Lépez I, L Furet \& O Aracena. 2001. Población de Emerita analoga (Stimpson 1857) en playas Amarilla y Rinconada, Antofagasta: aspectos abióticos, bióticos y concentración de cobre. Gayana 65: 55-76.

Margolis L, G Esch, JC Holmes, A Kuris \& G Schad. 1982. The use of ecological terms in parasitology (report of an ad hoc committee of the American Society of Parasitologists). Journal of Parasitology 68: 131-133. 
Mateo E, R Córdova \& E Guzmán. 1982. Polymorphus (Profilicollis) bullocki, nueva especie de acantocéfalo hallado en la gaviota Larus belcheri, en el Perú. Boletín de Lima 24: 73-78.

Mateo E, R Córdova \& E Guzmán. 1983. Polymorphus (Profilicollis) bullocki acantocéfalo de la gaviota Larus belcheri. Boletín de Lima 26: 67-71.

Nickol BB, Heard RW \& N Smith. 2002. Acanthocephalans from crabs in the Southeastern U.S., with the first intermediate hosts known for Arhythmorhynchus frassoni and Hexaglandula corynosoma. Journal of Parasitology 88: 79-83.

Oliva ME, J Luque \& A Cevallos. 1992. Parásitos de Emerita analoga (Stimpson) (Crustacea): implicancias ecológicas. Boletín de Lima 79: 77-80.

Oliva ME, I Barrios, $\mathbf{S}$ Thatje \& $\mathbf{J}$ Laudien. 2008. Changes in prevalence and intensity of infection of Profilicollis altmani (Perry, 1942) cystacanth (Acanthocephala) parasitizing the mole crab Emerita analoga (Stimpson, 1857): an El Niño cascade effect?. Helgoland Marine Research 62: 57-62.
Riquelme C, M George-Nascimento \& L Balboa. 2006. Morfometría y fecundidad de Profilicollis bullocki Mateo, Córdova \& Guzmán 1982 (Acanthocephala: Polymorphidae) en especies simpátricas de aves costeras de Chile. Revista Chilena de Historia Natural 79: 465-474.

Smith N. 2001. Spatial heterogeneity in recruitment of larval trematodes to snail intermediate hosts. Oecologia 127: 115122.

Smith N. 2007. Associations between shorebird abundance and parasites in the sand crab, Emerita analoga, along the California coast. Journal of Parasitology 93: 265-273.

Subramoniam T \& V Gunamalai. 2003. Breeding biology of the intertidal sand crab Emerita (Decapoda: Anomura). Advances in Marine Biology 46: 91-182.

Tantaleán M, J Cárdenas \& R Güere. 2002. Profilicollis altmani (Perry, 1942) Van Cleave, 1947 (Acanthocephala) en el Perú. Con notas sobre la infección experimental de mamíferos terrestres. Revista Peruana de Biología 9: 49-51.

Zar JH. 1984. Biostatistical analysis, 718 pp. Prentice-Hall, Englewood Cliffs.

Recibido el 21 de enero de 2010 y aceptado el 11 de mayo de 2010 